\title{
Foreword
}

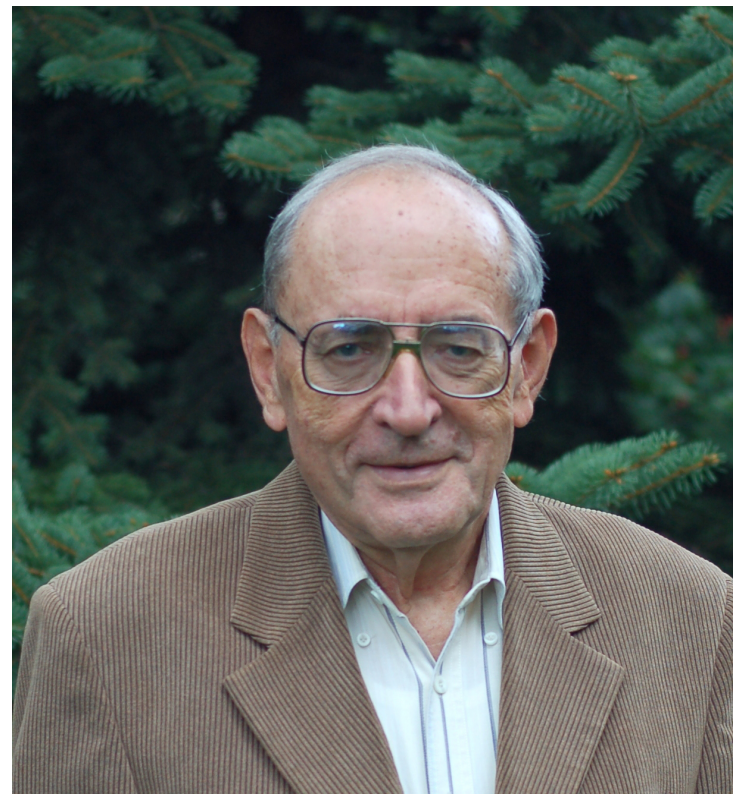

\section{Honorary issue dedicated to the 80th anniversary of Professor Ihor Stasyuk}

This special issue of the "Condensed Matter Physics" journal is dedicated to Professor Ihor Stasyuk, a prominent scientist and lecturer who celebrates his 80th birthday on September 23, 2018. The articles in this collections represent different fields of the quantum many-body physics and solid state theory, where Prof. I.V. Stasyuk made significant contributions and obtained remarkable results.

Ihor Stasyuk started his research work during his student years, when, together with his supervisor Professor Abba Glauberman and V.V. Vladimirov, he developed a "new form of polar model" and introduced the "site elementary excitation" operators - predecessors of the well-known Hubbard operators. These results were acknowledged by N.N. Bogolyubov and published first in Doklady Akademii Nauk SSSR [Soviet Physics — Doklady] in 1959 and later summarized in his Ph.D. thesis "The Method of Site Elementary Excitations in the Theory of Nonmetallic Crystals", which was successfully defended in 1963. Subsequently, he became an assistant professor at the Department of Solid State Theory and later at the Department of Theoretical Physics of the Ivan Franko State University of Lviv. During his time at the university, he obtained significant results in the theory of exchange interactions and ferromagnetism in strongly correlated electron systems, including development of the operator perturbation theory for models with local electron correlations and of Wick's theorem and diagrammatic techniques for Hubbard operators. Simultaneously, he initiated studies on the model description of dynamic and thermodynamic properties of complex hydrogen-bonded ferroelectric compounds and on the microscopic theory of optical effects in dielectric crystals.

In 1978, Ihor Stasyuk had to leave the Ivan Franko State University of Lviv and spent the next five years of his scientific activity connected with the Institute of Applied Problems of Mechanics and Mathematics of the Academy of Sciences of Ukraine, where he started working on electron-deformation effects in semiconductors and systems with narrow bands, as well as of crystals with a cooperative Jahn-Teller effect. At that time, he developed a unified microscopic theory of the optical effects induced by external fields in dielectric crystals, including electro-gyration and piezo-optic effect. 
In 1983, Ihor Stasyuk was invited to the Lviv Division of the Institute for Theoretical Physics (ITP) of the Academy of Sciences of Ukraine, now the Bogolyubov Institute for Theoretical Physics (BITP) of the National Academy of Sciences of Ukraine, where in 1985, he successfully defended his Doctor of Science (Habilitation) thesis entitled "Theory of External Field Induced Effects in Crystals with Structural Phase Transitions". In 1986, he became the Head of the Quantum Statistics Department. In 1990, the Lviv Division of the ITP was transformed into the Institute for Condensed Matter Physics (ICMP) of the National Academy of Sciences of Ukraine and starting from that time Ihor Stasyuk was the Research Deputy Director of ICMP until 2006. In 1995, Ihor Stasyuk was elected a Corresponding Member of the National Academy of Sciences of Ukraine.

Professor Ihor Stasyuk gained his most important scientific achievements in the field of condensed matter physics theory. He is widely recognized for developing mathematical methods of the theory of multilevel systems and for his research of fermionic systems with strong short-range correlations and physical phenomena in crystals with phase transitions. Professor Stasyuk investigates the effect of the Hubbard-type correlations and anharmonicity in the theory of high-temperature superconductivity and he is one of the authors of kinematic mechanisms of superconducting pairing in the Hubbard model. The school of Professor Stasyuk is widely acknowledged for the development of a microscopic theory for different types of field effects (hydrostatic and uniaxial pressure, electric field, single-ion anisotropy, etc.) on the properties and thermodynamics of ferroelectric and Jahn-Teller crystals. Another field of his interest is connected with the protonic and ionic transport in the systems with superionic phases. Recently, Stasyuk's interests concern the theory of intercalation induced effects in crystals, phase transitions and Bose-Einstein condensation in optical lattices.

Over his career to date, Prof. Stasyuk has authored five books and more than 700 scientific papers and contributions.

In addition to his research career, Professor Ihor Stasyuk is also well-known as an excellent teacher. He always combines his research work with the educational activities. Twenty one researchers under his supervision received Ph.D. degree; five of them became Doctors of Sciences. Numerous generations of students of the Ivan Franko National University of Lviv remember his brilliant lectures in theoretical physics, as well as his more specific courses in quantum statistics, electrodynamics, solid state theory, phase transitions theory, mathematical methods in theoretical physics, etc.

Professor Ihor Stasyuk is an active organizer of science. He is a full member of the Shevchenko Scientific Society, associate editor of "Condensed Matter Physics", editor of many international journals, member of the International Advisory Committee on Ferroic Domains and Mesoscopic Structures. For many years he was the vice-president of the Ukrainian Physical Society. Ihor Stasyuk has also served as either the conference chair or as a member of organizing and programme committee of many international and Ukrainian conferences in physics.

His scientific and public activity is acknowledged by many awards and titles. Professor Ihor Stasyuk is the Soros Professor (1996) and the "Excellence in Education of Ukraine" badge holder (1998). He was awarded the Certificate of Honour of the Supreme Council of Ukraine in 2004 and by the Insignia "For Scientific Achievements" of the National Academy of Sciences of Ukraine in 2008. In 2009 I.V. Stasyuk became the Chevalier of the Order of Merit of Third Class. In 2011 he was elected a Doctor Philosophiae Honoris Causa at the Bogolyubov Institute for Theoretical Physics of the National Academy of Sciences of Ukraine and in 2014 he was awarded the A.S. Davydov Prize of the National Academy of Sciences of Ukraine.

The Editors of this special issue, the Editorial Board of "Condensed Matter Physics", and his numerous colleagues and friends congratulate Professor Ihor Stasyuk on the occasion of his anniversary and acknowledge his unique and valuable contributions to science. We wish him to stay in a good health and to continue his work for many more enjoyable and productive years. We also express our gratitude to all authors who contributed their papers to this anniversary edition.

Jim Freericks (Department of Physics, Georgetown University, Washington, DC, USA) Andrij Shvaika (Institute for Condensed Matter Physics of the National Academy of Sciences of Ukraine, Lviv, Ukraine) 